\title{
The Innateness Hypothesis and Mathematical Concepts
}

\author{
Helen De Cruz and Johan De Smedt
}

This is the final draft of the following paper: De Cruz, H., \& De Smedt, J. (2010). The Innateness Hypothesis and Mathematical Concepts. Topoi, 29(1), 3-13.

http://www.springerlink.com/content/q36005807h158v32/?p=2c759fbbfc6e4a5891e9a2 2d43743f9c\&pi=1

\begin{abstract}
In historical claims for nativism, mathematics is a paradigmatic example of innate knowledge. Claims by contemporary developmental psychologists of elementary mathematical skills in human infants are a legacy of this. However, the connection between these skills and more formal mathematical concepts and methods remains unclear. This paper assesses the current debates surrounding nativism and mathematical knowledge by teasing them apart into two distinct claims. First, in what way does the experimental evidence from infants, nonhuman animals and neuropsychology support the nativist hypothesis? Second, granting that infants have some elementary mathematical skills, does this mean that such skills play an important role in the development of mathematical knowledge?
\end{abstract}

\section{Nativism and mathematical knowledge}

Until the late 18th century, mathematical knowledge was a paradigmatic example of innate knowledge. It often served a dual purpose, providing both a persuasive reason why innate knowledge exists, and furnishing us with an explanation of how humans can have knowledge of mathematical objects. Due to the increasing importance of foundational issues and the explicit rejection of psychologism (i.e., the view that mathematics lies within the purview of psychology) by Frege and others, nativism has lost its importance in recent philosophy of mathematics. By contrast, contemporary developmental psychologists (e.g., Spelke, Kinzler, 2007; Carey, 2004; Feigenson et al., 2004) propose that innate ideas are a naturalistic source of mathematical knowledge. They have uncovered evidence for earlydeveloped mathematical skills in infants and young children, such as the ability to estimate and discriminate numerosities (Jordan, Brannon, 2006), the capacity to detect ordinal relationships between numerosities (Brannon, 2002), and the ability to reason about spatial relationships in a Euclidean framework (see De Cruz, 2009, for a review).

This article examines the relevance of nativism for the philosophy of mathematics. After providing a brief overview of historical claims for innate mathematical knowledge, we focus on arithmetic as an example of innate mathematical knowledge in current developmental psychology. We examine in detail the claim that infants are capable of predicting the outcomes of simple 
addition and subtraction events, and look at recent objections to it. Then, we examine to what extent this innate knowledge is important for mathematical practice. Focusing on number theory and arithmetic, we find that there are three possible ways to explore the relationship between innate ideas and mathematical practice.

\subsection{Historical claims}

Plato's Meno (ca. 380 BC [2000]) constitutes one of the earliest direct treatments of the innateness hypothesis, and interestingly, it draws extensively on a mathematical example. In this dialogue, Socrates probes the geometric intuitions of an uneducated slave boy, leading him through a series of questions to discover relationships between the areas of squares drawn in the sand, including a reiteration of the Pythagorean theorem. Given that the boy did not learn geometry during his lifetime, Plato concluded that the slave must have always possessed this knowledge, and that our learning of geometric concepts is actually recollecting (anamnesis) of what we have always known as immortal souls. Descartes (1637 [1988], AT VI 135-137) proposed that our experience is too limited to generate mathematical concepts such as TRIANGLE, as we are always confronted with imperfect examples. He also believed mathematical intuitions to underlie more mundane forms of reasoning, for example, that stereoscopic vision is possible through une géometrie naturelle, an innate geometry that allows us to combine the two-dimensional images from our eyes into a three-dimensional representation of space. Leibniz (1765 [2001]) argued that our experience of the world is always of contingent particulars, but that our knowledge can be general, and sometimes necessary - this is especially the case for mathematics. He recognized that, while our knowledge of numbers is learned, the cognitive capacity that enables us to learn them is innate: "And I cannot accept the proposition that whatever is learned is not innate. The truths about numbers are in us; but still we learn them" (Leibniz, 1765 [2001], 85). The potential knowledge of necessary mathematical truths is thus innate, comparable to the veins of a marble that outline a shape within it before being uncovered by a sculptor, just like learning can uncover our innate knowledge (Leibniz, 1765 [2001], 52). Kant's argument from geometry presents perhaps the most intricate classical philosophical claim for the innateness of mathematical intuition. Kant (1781 [2005], A25/B40) specifies that our intuition of space does not and cannot be derived from outward experience, since the sensation of experiences outside of the self already requires that one possesses the concept SPACE. Then, he goes on to argue that it is precisely this intuition of space that enables us to develop geometry as a scientific discipline. Since Euclidean geometry requires us to determine properties of space synthetically and a priori, the intuition of Euclidean principles must already exist within the subjects who learn this discipline (Kant, 1781 [2005], B41).

The argumentative structure of historical accounts of innate knowledge is very similar to that of scientists working within nativist research programs today. Like historical nativists, modern cognitive scientists invoke poverty of the stimulus 
arguments: given that our experience is too limited to generate mathematical truths, our knowledge of those truths must already be contained within us. Ever since Chomsky's seminal work on language development in children, innateness is a central concept in cognitive science. Yet the notion of 'innate' remains ill defined, such that some philosophers of science (e.g., Mameli, Bateson, 2006) have argued that the innate/acquired distinction is incoherent and should be abandoned altogether (for one thing, all traits are acquired at some point during the development of an organism from fertilized egg cell to mature individual). By contrast, others (e.g., Samuels, 2004) are not concerned with defining the meaning of the term 'innate', but attempt to explain its role and significance within scientific practice. Despite its lack of an unambiguous definition, cognitive scientists continue to use the concept of innateness in their explanatory frameworks, and, as we shall see, the case of mathematics continues to play a role in current investigations of innate knowledge.

\subsection{Current positions in philosophy of mathematics}

The question of how humans are able to get epistemic access to mathematical objects is still relevant today. Benacerraf (1973) famously argued that causal epistemic accounts of mathematical knowledge are problematic, because mathematical objects are often characterized as abstract entities, which reside outside of space-time, making it difficult for mathematicians to acquire knowledge about them. Few contemporary philosophers of mathematics are attracted to innateness as a possible solution to this problem. To take but one example of a recent response to Benacerraf, according to Shapiro's (1997) ante rem structuralism, non-applied mathematics is concerned with structures that are conceived of as abstract entities. The precise nature of these abstract entities is left unspecified, as it is not essential to mathematical practice. Just as one can talk about a goal keeper's function in soccer (i.e., keeping the ball out of the goal) without going into detail about the precise properties of the person in this position (e.g., hair color), a mathematician can talk about the natural number 2 as a position within the structure of arithmetic without having to worry about which settheoretical conceptualization captures 2 best, such as $\{\varnothing,\{\varnothing\}\}$ or $\{\{\varnothing\}\}$. If mathematical knowledge can indeed be derived from structural properties, there is no need to look for an experiential basis of mathematical knowledge. However, Shapiro still needs to explain how we can conceive of structures in the first place, and how we can grasp what is true about them. In response to this, Shapiro (1997, 115) invokes our ability to recognize patterns and to abstract from particulars, leading us to recognize, for example that "the four pattern is the structure common to all collections of four objects." A similar account is Resnik's (1982) 'experiencing something as patterned', where mathematics is conceived of as the study of patterns. However, this capacity, pattern recognition, remains to be explained. Shapiro's $(1997,115)$ explanation is not psychologically satisfying: "[the] child starts to learn about cardinal structures by ostensive definition. The parent points to a group of four objects, says "four", then points to a different 
group of four objects and repeats the exercise. Eventually, the child learns to recognize the pattern itself". It remains to be explained how the child can recognize what it is that stays invariant in the different sets presented to her (i.e., cardinality). At some point, humans must be able to discriminate cardinality, and it is not clear how children can accomplish this. How do we learn to abstract the four pattern from four notes played on a keyboard, four trucks passing by or four TV commercials, without already having some notion of what cardinality is?

\section{Innate mathematical knowledge in developmental psychology: the case of arithmetic}

Contemporary scientists working within a nativist research program consider mathematical knowledge as a paradigmatic example of innate knowledge (e.g., Spelke, Kinzler, 2007). They rely on a special version of the poverty of the stimulus argument which Samuels (2002) calls 'the argument from early development'. According to this, a given concept emerges at a point when it could not have been learned through experience.

\section{The argument from early development}

1. Under experimental conditions, it is observed that infants possess a certain capacity, for example, infants from a few hours after birth can visually discriminate between collections of two and three objects (Antell, Keating, 1983).

2. This capacity is seen as best explained by positing some conceptual capacity, in this case, a rudimentary ability to discriminate small sets.

3. Because the capacity arises so early in development, it could not have been learned through experience, i.e., newborns, having spent time in the dark environment of the womb, did not have the opportunity to learn to visually discriminate sets with different numbers of items.

4. Hence, the structure in question (discrimination of small numerosities) is probably innate.

To give a focus to our discussion of innate mathematical knowledge, we will look at one case in detail, namely the question of whether or not infants possess an innate capacity to perform arithmetic. In 1992, developmental psychologist Karen Wynn published an elegant study in which she argued that our ability to perform the simple arithmetic operations of subtraction and addition is already present in five-month-old infants. Her experiment was based on the violation of expectation paradigm, a procedure that is frequently used to probe infants' knowledge. It relies on the assumption that infants look longer at events that they do not expect because these events are more interesting to them, a property of the human perceptual system that magicians rely on to capture the attention of their audience. 
Infants are first habituated to a given event, which is repeated over and over until their attention wanes. Then, during the test itself, subjects are randomly assigned to a control condition, or to a condition in which the event is slightly modified so that it violates expectations. With appropriate controls, evidence that infants look reliably longer at the unexpected than at the expected event is taken to indicate that they (1) possess the expectation under investigation; (2) detect the violation in the unexpected event, and (3) show an increased interest for this violation. In Wynn's experiments, infants witnessed how a puppet was placed on a stage. A screen was lowered, hiding the puppet, and they saw how a second doll was placed behind the screen. If infants can perform the addition $1+1=2$, they should expect to see two objects once the screen is lowered. Indeed, Wynn (1992) found that infants looked longer when only one puppet was visible $(1+1=1)$. Similarly, infants who saw two puppets on the stage, a screen placed in front of them, and one of the puppets being taken away, looked longer at the event $2-1=2$ than at 2 $-1=1$, which Wynn interpreted as evidence that they can perform simple subtractions. More recently, McCrink and Wynn (2004) repeated this experiment with larger numbers: infants were either presented with possible arithmetic operations $5+5=10$ and $10-5=5$, or impossible results $5+5=5,10-5=10$. Again, the five-month-olds looked longer at the impossible results.

These experiments can be mapped onto the argument from development as outlined earlier. First, a difference in looking time between correct and incorrect outcomes is observed (premise 1). This difference in looking time is attributed to a capacity of the infants to discriminate between correct and incorrect arithmetic operations (premise 2). The time span of five months is deemed to be insufficient to enable infants to learn to perform arithmetic operations through experience (premise 3). Hence, the capacity is attributed to the innate ability to discriminate between correct and incorrect arithmetic operations (conclusion). "[I]nfants possess true numerical concepts - they have access to the ordering of numerical relationships between small numbers and can manipulate these concepts in a numerically meaningful way [...] The existence of these arithmetical abilities so early in infancy suggests that humans innately possess the capacity to perform simple arithmetical calculations, which may provide the foundations of further arithmetical knowledge" (Wynn, 1992, 750). Thus, like in the early philosophical arguments, mathematical knowledge is taken to be innate, and to serve as a basis for the development of further mathematical skills. The argument from early development relies on a chain of inductions. The quality of each element in this chain determines the validity of the argument. Its serial nature also means that one flaw can potentially undermine the entire argument. To examine whether the conclusion is cogent, we will now look at some objections that have been raised against each step in the chain.

\subsection{Premise 1: infants' looking time differs between correct and incorrect arithmetic operations}

One possible way to call experiments like these into question is to doubt the 
experimental procedure on which they are based. Experiments with infants and animals are susceptible to the so-called Clever Hans effect, named after an early $20^{\text {th }}$-century horse that could allegedly solve arithmetic operations by tapping the correct answer with its hoof. After careful examination, psychologists found that Hans was sensitive to unconscious cuing of the audience or its trainer. Ever since then, many precautions are taken to eliminate Clever Hans effects in animal studies and developmental psychology. Infants are either seated in a car seat, or placed on the lap of their parents who wear dark goggles so as not to see the experimental conditions. The looking times of infants are coded by two independent observers who cannot see the experimental procedure, i.e., they do not know when it switches from habituation to test, and they do not know whether the infants are watching the unexpected or the expected event. Statistical measures of interobserver agreement are made; only results with a high interobserver agreement are taken into account. Thus it is unlikely that the experimenters or parents influence the judgments of the infants. The experimental setup, and the observed differences in looking time are therefore usually accepted, also by opponents of nativism.

\subsection{Premise 2: this success is best explained by the infants' conceptual knowledge of number}

This step is the most hotly debated by psychologists who prefer alternative empiricist explanations. For example, Haith (1998) considers a simpler perceptionbased alternative: in the case where $1+1=1$, infants are surprised because one of the objects disappears. If infants still have lingering perceptual information about the one doll prior to the screen being raised, next to that of the doll that is being placed behind it, they should expect 2 dolls. This causes a mismatch between the subjects' purely perception-based expectation and the actual situation (1 doll). Hence, longer looking-times are caused by extremely long-term sensory persistence of each object prior to its occlusion-the infants will look longer at $1+1=1$ without any arithmetic skills. A problem with this alternative explanation is that it invokes a novel mechanism, long-term sensory persistence. As there is no empirical evidence of its existence, it would seem that this mechanism is invoked with the sole purpose of providing a non-cognitive account of these experiments. Nonetheless, some replications of Wynn (1992) have attempted to eliminate this alternative account. Koechlin et al. (1998), for example, replicated the experiment with the puppets on rotating platforms; the constant revolving of the puppets across the stage excludes the possibility of extreme sensory persistence. Even under these conditions, infants looked longer when they witnessed impossible outcomes of subtractions and additions.

Explaining the results in terms of a familiarity preference might be a more promising non-cognitive explanation. According to Cohen and Marks (2002), infants look longer at $1+1=1$ because they see only one doll at the beginning of the experiment, when they are familiarized with the setup by being seated in front of the theatre and watching one doll. Similarly, they prefer $2-1=2$ because during these familiarization trials they more frequently see two dolls. To test this 
alternative explanation, Cohen and Marks (2002) designed a series of experiments in which infants were shown $0,1,2$, and 3 as outcomes of $1+1$ and $2-1$ operations. The results were consistent with their interpretation of the evidence: the infants looked much longer at $1+1=1$ and $2-1=2$ than at the other incorrect outcomes $(1+1=0$ or 3 and $2-1=0$ or 3$)$. To control for this bias, Kobayashi et al. (2004) replicated Wynn's experiment across modalities (visual and auditory). In their experiments, five-month-olds were shown a computer-animated version of Wynn's experimental setup. Familiarizations consisted of 1, 2 or 3 dolls falling onto a platform, producing a distinctive thud with each fall. In the $1+1=2$ or 1 condition, infants watched a doll falling from the top of the display onto a platform, making a distinct thud. After this, a screen appeared in front of the doll, and the subjects heard a second, similar thud. After the screen was lowered, they looked longer when only one doll was present. Similar results were obtained for the subtraction condition. Since this study controlled for possible familiarity preferences, it indicates that familiarity alone cannot explain the experimental results. Berger et al. (2006) replicated Wynn's original setup: six- to nine-montholds saw incorrect and correct outcomes while their brain activity was measured by electrodes placed on the scalp (a procedure known as Event Related Potentials). In the adult human brain, there are well-described patterns of brain activation associated with error detection. The infants' brains, like those of adults, showed the same characteristic pattern of error-detection during the incorrect outcomes. This provides additional support for the cognitive interpretation of Wynn's experiments. It appears that there is currently no rival empiricist explanation that accounts for the results of these experiments.

\subsection{Premise 3: because the capacity arises early in development, it cannot have been learned through experience}

One can never state with absolute certainty that an early-developed skill is not the result of experience, except for skills that are tested immediately after birth, like face recognition. After all, as Haith (1998) observed, infants of a few months old have had over 1000 hours of waking time, corresponding to millions of eye movements, which could have provided plenty of opportunity to benefit from visual experience. However, in the case of arithmetic visual experience alone seems insufficient. Five-month-old infants cannot voluntarily grasp and release objects: grasp is present at 4 to 5 months, but voluntary release only at 9 months due to persistence of the grasp reflex. Empiricist accounts of the acquisition of arithmetical skills such as that by Piaget (1952) propose that young children learn the outcomes of arithmetic operations by placing objects together, or taking some away, and observing the resulting number of objects. Infants of five months old, who can barely grasp and not release, cannot experiment in this way by adding or taking away objects to predict the results of additions and subtractions. Of course, this does not rule out other possible empirical ways in which infants could have learned to predict the outcome of arithmetic operations. It may well be that whether one takes an empiricist or nativist perspective to interpret a given body of 
evidence depends on the judgment (in the Kuhnian sense) of the experimenter, rather than an unambiguous reading of the evidence. Without a detailed empiricist rival explanation, nativist developmental psychologists may be justified in claiming that these experiments provide evidence for innate arithmetic knowledge, without excluding the possibility of a future empiricist rival account.

\subsection{Conclusion: the property in question is probably innate}

What does 'innate' mean? Long before innateness was a scientific concept, it was a philosophical and a folk concept. The historical example of the folk concept of FORCE that was imported into physics illustrates that such altered folk concepts can and do play a legitimate role in science (Mameli, Bateson, 2006, 156). The folk concept of innateness is currently being imported into sciences as diverse as cognitive science, embryology and palaeoanthropology, where it gradually changes to fit the purposes of these disciplines. Because innateness has been imported into many diverging disciplines, the explanatory role it assumes within these differs. As a result, there is no general definition of 'innateness' that covers all disciplines. Evolutionary psychologists, for example, regard an innate trait as an evolutionary adaptation. They place an emphasis on universality and developmental invariance, e.g., cross-cultural features of human mate selection are seen as evidence for evolved preferences for desirable traits in mates. By contrast, developmental psychologists do not use explicit evolutionary frameworks to reason about knowledge in infants. Rather, they consider cognitive traits to be innate if there is no correct developmental psychological account to explain how the infants could have learned them. This is precisely the motivation behind the argument from early development. Because the explanation of innateness depends on the discipline in which it is used, Samuels (2002) regards an innate idea as one for which there is no correct scientific account within that discipline. In the case of innate arithmetic, if developmental psychologists cannot explain this ability as a result of learning, it becomes 'innate' by default within that discipline. This is not to say that there is no correct scientific account for this ability in another discipline, such as genetics or cognitive neuroscience.

\section{From intuitive to formal mathematical knowledge}

If nativist claims for mathematical principles turn out to be sound, how can we assert that these skills are important for the development of mathematical knowledge? Although both claims (i.e., mathematical skills are innate, and these innate skills form the basis of mathematical knowledge) are logically independent, they are often conflated. Until recently, developmental psychologists assumed almost without question that the rudimentary numerical capacities exhibited by infants and nonhuman animals lie at the basis of more complex mathematical knowledge. Nevertheless, the question of how these capacities give rise to mathematical theory remains unresolved.

A wealth of empirical evidence suggests that the capacities to represent 
numerosities, to perform elementary arithmetic operations, and to detect ordinal relationships between different collections of items are present in infants and nonhuman animals (see Feigenson et al. 2004, for an overview). It seems reasonable to suppose that such capacities form the precursors of formalized ways of reasoning about number and arithmetic. But it remains difficult to pinpoint the causal arrow between intuitive and formal mathematical concepts. It is quite possible that both domains are independent, i.e., that there is no overlap between intuitive and formal mathematical concepts, and that the similarities are only superficial. In order to reconcile intuitive and formal numerical competence, three possible ways are open to us. One strategy consists in attempting to characterize preverbal numerical representations in terms of number theory. If intuitive number concepts are in some way isomorphic to theoretical number concepts, then it is conceivable that children can go from their initial numerical skills to a understanding of natural numbers, negative numbers, fractions and so on. A second strategy examines the process of natural number acquisition in young children, and attempts to spot parallels between this process and properties of number theory -if such parallels exist, it is relatively unproblematic to explain the emergence of formal number concepts in children. A third strategy is more indirect, and attempts to show that intuitive number concepts are important for the acquisition of more formalized arithmetic skills. This approach can be situated within philosophical positions that emphasize mathematical practice, rather than the theoretical foundations of mathematics. We will critically discuss each position in turn.

\subsection{Characterizing intuitive numbers formally}

How can intuitive numerical representations be characterized? Several mathematicians (e.g., Kronecker and Brouwer) accorded a privileged position to the natural numbers in their attempt to find a foundation for arithmetic: they thought that natural numbers were given through intuition, and that from these we can construct all other numbers. However, there is a mismatch between the natural numbers and the representations of cardinality by nonhuman animals and human infants. The latter are imprecise; this imprecision increases rapidly with the magnitude of the numerosities. For example, while newborns can reliably discriminate between collections of two and three items, they fail to see the difference between four and six, although the ratio difference is identical (Antell \& Keating, 1983). Likewise, in a classical study (Meck, Church, 1983) in which rats were trained to press a lever $n$ times (with $n$ ranging between 4 and 24), the animals became less and less accurate as the required number of lever-presses increased. The same increasing imprecision is also observed in adults who are required to make a number of key presses or to estimate the number of light flashes at a rate that makes counting impossible (Whalen et al., 1999).

To date, no nonhuman animal has mastered the open-ended structure of natural numbers in a way that four-year-old human children in many cultures have. Typically, chimpanzees and other animals can be taught to remember numerosities 
by learning each magnitude separately. In one study, a female chimpanzee learned to associate arabic digits with cardinal values by brute association. In the initial training of the number 2 , she apparently assumed that ' 2 ' meant 'more than one', eventually learning to apply the arabic numerals ' 1 ', ' 2 ' and ' 3 ' correctly. Instead of generalizing this procedure to numbers greater than 3 , she evidently went on to assume that ' 3 ' meant more than two, which brought her competence at assigning '4' correctly to chance level until she eventually also learned 4, and this up to 9 (Biro, Matsuzawa, 2001). The insight that this property can be generalized, i.e., that for every natural number $n$ there is a number $n+1$, appears to be restricted to humans.

Interestingly, several human cultures seem to lack natural number representations. For example, the Pirahã, a Brazilian indigenous culture, do not possess words that denote natural numbers (Gordon, 2004). The Pirahã language has only three standardized words to denote cardinality, often translated as 'one' (hói), 'two' (hoí) and 'many' (baágiso). In one study (Frank et al. 2008), Pirahã subjects were presented with arrays of objects in increasing order (from 1 to 10) and asked to denote how many items they saw. Subsequently, the items were presented in decreasing order (from 10 to 1). The use of the terms 'hói', 'hoí' and 'baágiso' was not consistent, but depended on the order in which the items were presented: when objects were presented in increasing order, most subjects said that 6 items were 'baágiso' (many), whereas in decreasing order, most subjects said 'hoí' (two) when presented with the same 6 objects. Hence, the authors concluded that the Pirahã language has no words that correspond to our concept of natural numbers, not even in an approximate sense. Given that natural numbers are not present in infants and nonhuman animals, and not even in all human cultures, they are unlikely candidates to describe our innate numerical skills.

Gallistel and Gelman (2000) proposed that magnitudes on the mental number line could be conceptualized as real numbers. Real numbers differ from natural numbers in several interesting respects: the latter are countable and discrete, whereas the former (e.g., $\sqrt{ } 2$ or $\pi$ ) are uncountable and dense; they cannot be put into a one-to-one correspondence with a list of items. Gallistel and Gelman chose reals, because they are non-discrete like the representations of number in infants and nonhuman animals. As shown in figure 1a, these can be illustrated by tuning curves, which are normally distributed around given quantities. In the rhesus monkey brain, individual neurons that respond to numerosity show this approximation: neurons optimally responding to 4 items also exhibit some activation for values between 2 and 6 (Tudusciuc, Nieder, 2007). However, there is no evidence that nonhuman animals would be capable of representing real numbers such as $e$ or 1.01001000100001. Moreover, as natural numbers form a subset of the real numbers, this account fails to explain why animals are not able to master natural numbers. It remains unclear how humans go from this kind of numerical concept to counting: in contrast to natural numbers, there is no definite next tag within real numbers, e.g., for 1 , any number greater than 1 such as 1.00000000001 could be a next tag (Laurence, Margolis, 2005).

Whereas the set of natural numbers can be mapped onto a linear scale, it 
remains unclear whether or not animals and infants represent numerosities in this way. Their representations appear to be compressed for larger magnitudes such that the perceived distance between 2 and 3 is larger than the perceived distance between 22 and 23. This effect of decreasing discriminability with increasing size is very robust, and has been documented in nonhuman animals (Meck, Church, 1983), preschoolers (Siegler, Booth, 2004), and in people from cultures with inexact number words (Dehaene et al., 2008). Hence, some authors have proposed that such estimations of numerosities conform to the natural logarithms ( $L n)$ of these numbers (Figure 1a). As can be seen, the variability is fixed, i.e., around each value the variability of responses follows the same normal distribution. As the logarithmic curves overlap increasingly with their neighbors, higher numerosities are more difficult to tell apart - this explains why it is easier to tell 3 items from 4 than it is to discriminate 23 from 24 .
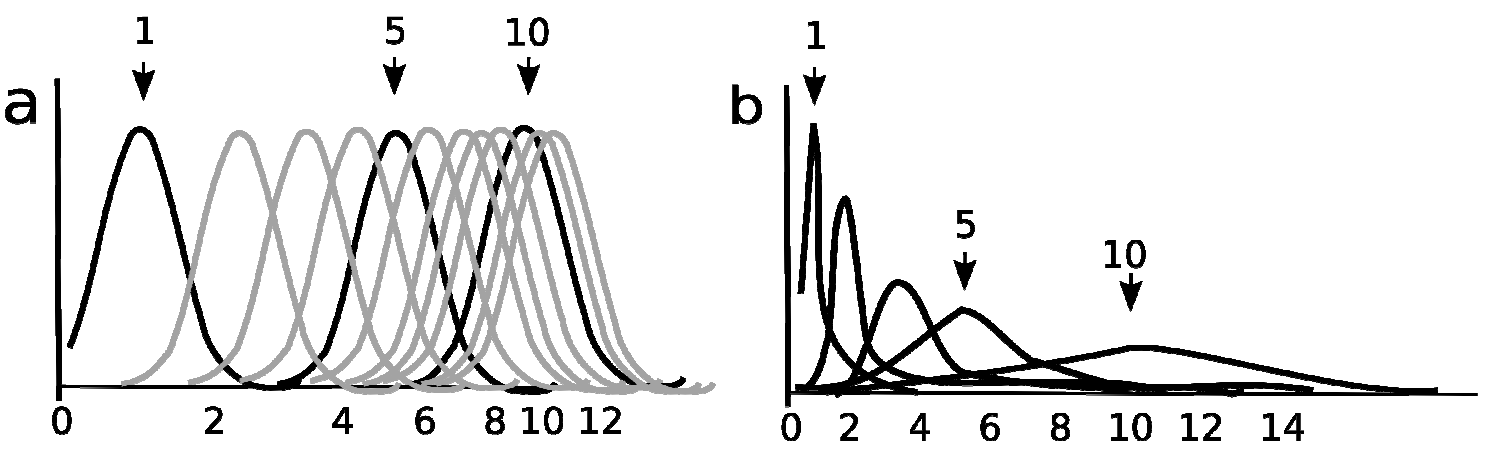

Fig. 1: Two competing hypotheses on how intuitive numerosities are represented (a) logarithmic with fixed variability, and (b) linear with scalar variability

However, several psychologists (e.g., Le Corre, Carey, 2007) disagree with this interpretation, and contend that intuitive number representations, both in young children and nonhuman animals, can be more accurately captured using a linear representation with scalar variability (Figure $1 \mathrm{~b}$ ). Scalar variability means that the standard deviation of the estimate of some quantity is a linear function of its absolute value, i.e., the larger the value, the higher the standard deviation, and the broader the tuning curves. Therefore, representations of small collections up to 3 or 4 are reasonably accurate, whereas those of larger numbers become increasingly imprecise and noisy. It remains difficult to decide between logarithmic and scalar variable representations because they yield similar predictions in most (but not all) circumstances. For example, they both predict the distance effect, namely the fact that smaller numerosities are discriminated faster and more accurately. In sum, the evidence taken together suggests that intuitive number representations do not correspond to any well-established set in number theory (such as natural numbers or reals), and there is currently no consensus as to how they can be best represented (logarithmic or linear). Given the current state of affairs, attempts to 
characterize preverbal number concepts in terms of number theory reveal little about the relationship between intuitive and formal mathematical ideas.

\subsection{Learning natural numbers through axiomatic systems}

Some philosophers of mathematics focus on how children learn the natural numbers. If this learning process captures some significant properties of axiomatizations of arithmetic, such an approach might highlight important psychological continuities between intuitive and formal concepts. According to one account, children learn the natural numbers by adopting the successor function, which is central to the Dedekind-Peano axiomatizations of arithmetic. The successor function is a primitive function, which states that if a given $n$ is a natural number, so is its successor, i.e., $S(1)=2, S(2)=3$, and so on. Many psychologists (e.g., Le Corre, Carey, 2007) regard the successor function as a crucial building block in the understanding of numerosities. According to one popular account (e.g., Carey, 2004), children learn to map the meanings of the words for 'one', 'two' and 'three' onto their pre-existing intuitive representations of these quantities. This seems sensible, as nonhuman animals can also easily discriminate between these small collections (e.g., Uller et al., 2003). Children then recognize the successor function within the first words of their counting list, and, through induction generalize this principle to higher number words (four, five, and so on): if a numeral $n$ refers to cardinal value $n$ and $p$ immediately follows $n$ in the count list then $p$ refers to $n+1$. A problem with this account is that the Dedekind-Peano axioms do not characterize our everyday use of number words. Some authors (e.g., Rips et al., 2008) are skeptic about the assumption that children can make the inductive step from small numerosities to a list of counting words. For one thing, why would children conclude that 'two' refers to collections of exactly two objects, rather than approximately two objects, as the Pirahã do? As we have seen, nonhuman animals, even our closest relative the chimpanzee, seem to be unable to do so, even after extensive training (Biro, Matsuzawa, 2001).

Decock (2008) provides an alternative account in which equinumerosity (also known as Hume's principle) rather than the successor function is conceptualized as the basis for learning natural numbers. According to this notion, if humans have the concept of COLLECTION, they can discover that two collections have the same number of items by putting their members into a one-to-one correspondence, either physically or by means of a stably ordered verbal list of counting words or body parts. Indeed, in many cultures, one-to-one correspondence is used to denote quantities in this way. The Loboda, for example, are a Papua New Guinean aboriginal culture where gift exchange plays an important role in the local economy. To judge whether a gift is fairly distributed or reciprocated, objects of a specific category (e.g., yams, bunches of tobacco) are piled up or placed in baskets, and these collections are compared to each other. Once the collections are of equivalent size, the Loboda know that the distribution is fair, even though they 
do not know the exact cardinality (Thune, 1978). Also, some experiments (e.g., Jordan, Brannon, 2006) suggest that infants can spontaneously detect equinumerosity: they can match the number of talking heads they see on a screen to the number of voices they hear. Still, Hume's principle, as Rips et al. (2008) note, is not sufficient to specify the natural numbers. It is consistent, for example, with systems containing only a finite set of numbers, and with systems that contain cardinals beyond the natural numbers (e.g., fractions). To get positive integers only, one needs to invoke some additional definitions that require successor series.

Both approaches share a similar problem. Even if the successor function or equinumerosity can provide a basis of arithmetic from a formal point of view, this does not guarantee that either actually lies at the basis of numerical abilities. These axiomatizations were never meant to conceptualize everyday numerical skills, but rather to provide a consistent foundation from which arithmetic could be derived. As Dedekind (cited in Greiffenhagen, Sharrock, 2006) remarked: "...many a reader will scarcely recognize in the shadowy forms which I bring before him his numbers which all his life long have accompanied him as faithful and familiar friends." Conflating number theory with everyday practices of counting may be a category mistake.

\subsection{The importance of innate numerical skills for mathematical practice}

Everyday mathematical practice suggests a distinction between the foundational work of axiomatizing mathematical theory and the daily work of mathematicians. Dedekind noted that axiomatizations of arithmetic are not meant to capture our everyday use of number; it is also interesting to observe that mathematicians rely to an important extent on informal, intuitive modes of reasoning, especially in the early stages of creativity. In their description of the mathematical experience, Davis and Hersh $(1981,399)$ stress the importance of intuitive, everyday mathematical practice, and go as far as to say, "[T] he study of mental objects with reproducible properties is called mathematics. Intuition is the faculty by which we can consider or examine these (internal, mental) objects". In this way, everyday numerical practice, such as arithmetic or comparing numerosities, is continuous with formal mathematics. Indeed, the emphasis on formal aspects of mathematics, such as proofs, is a recent phenomenon of western culture that seems absent in other cultures with a rich mathematical tradition, like the medieval Islamic world or imperial China. Even in western mathematics up to the $18^{\text {th }}$ century mathematicians were primarily concerned with getting results, and their successes were many. It is doubtful whether these successes would have been achieved had Euler and his contemporaries been burdened by the standards of rigor of today. In the early $19^{\text {th }}$ century, this situation changed dramatically: mathematicians such as Cauchy and Bolzano demanded rigorous proofs of the theorems about concepts that had hitherto been understood mainly intuitively. For example, as early as 1629 , Girard wrote that any $n$th degree equation has $n$ real roots-a first step towards what later became known as the Fundamental Theorem of Algebra. 
However, it was not until 1806 that Argand published a rigorous proof of it, for the first time specifying that the theorem holds when the coefficients are complex, rather than real (Grabiner, 1986). Still, the role of nonformal mathematical practice has not disappeared in current mathematics. The initial belief that a proof may be correct, such as Wiles' proof of Fermat's last theorem, usually does not depend on thorough scrutiny, but on concurrence with high-level ideas (some might say, gut feeling) long before the details are checked (Thurston, 2006).

Another reason to think that informal mathematical practice is important for the development of mathematics as a formal discipline is historical. Whenever accurate historical accounts of mathematical practice are available, we can observe that mathematical techniques develop in response to practical needs. The elaborate mathematical tools developed in Han dynasty China (202 BC-220 AD), which involved solutions to simultaneous linear equations with several unknowns, was concerned with the needs of the early developing empire, and provided solutions for calculating taxes, dividing inheritance, and organizing large-scale public works. Medieval Islamic geometry was applied to prestige architecture (e.g., the construction of a round cupola on the square basis of mausoleums), seafaring and the calculation of the q'ibla, the relative position of Mecca. If it can be shown that innate mathematical abilities are indeed essential for the development of mathematical practice, then we have reason to believe that these are in some way foundational for mathematics as a formal science, as the early nativist philosophers like Plato, Descartes, Leibniz and Kant originally proposed.

To illustrate this line of reasoning, we again draw from the domain of arithmetic. As we have argued in section 2, infants are able to predict the outcomes of very simple arithmetic operations, but this does not imply that this ability underpins the later development of more complex arithmetic skills. Yet several lines of evidence suggest that innate arithmetic skills are constitutive of later arithmetic competence. Gilmore et al. (2007) asked five-year-olds to solve symbolic arithmetic tasks in verbal format or with arabic digits. The problems involved large numbers, such that the preschoolers could not have learned the outcomes yet, for example "Sarah has fifteen candies and gets nineteen more, John has fifty-one candies. Who has more candies?" Children had about $73 \%$ of the answers correct, significantly above chance, which rules out guessing as a strategy. The authors then examined possible reasons for the children's successes. First, they looked at the possibility that they might have learned some symbolic arithmetic facts by heart (e.g., $5+9=14$ ) and drew on these as a basis for other problems. However, the subjects failed to provide correct solutions to exact problems of this form. Next, they examined whether or not children drew on approximate arithmetic. They found that the accuracy depended on the ratio between the numbers, a clear signature of approximate numerical competence, which has also been found in monkeys-when rhesus monkeys perform arithmetic tasks, they also become increasingly imprecise as the ratio difference drops (Flombaum et al., 2005). In agreement with the view that approximate arithmetic skills underlie exact arithmetic performance, Halberda et al. (2008) showed that teenagers who do well 
on approximate arithmetic tasks are more likely to perform well on mathematics at school.

Studies that measure differences in brain activation (e.g., Venkatraman et al., 2005) indicate that nonsymbolic arithmetic activates brain regions that are very similar to those involved in symbolic arithmetic. Regardless of whether subjects solve additions by looking at collections of dots that are added together or by adding up arabic digits, the intraparietal sulci, areas of the brain involved in a wide variety of numerical tasks, show increased activation. Previous studies that measured the individual activation in neurons of rhesus monkeys (Tudusciuc, Nieder, 2007) indicate that the intraparietal sulci contain number-sensitive neurons, which are sensitive to the cardinality of a collection of items, but not sensitive to other properties, such as size or shape. Although these studies do not demonstrate a direct, causal link between innate and formal mathematical knowledge, they suggest that innate abilities are essential for the development of mathematical skills.

If approximate numerical competences are important for the development of formal mathematics, one would expect that people from cultures without formal arithmetic can spontaneously figure out arithmetic principles. The Yupno, a Papua New Guinean aboriginal people, do not perform arithmetic operations, possibly because it has little practical value for them. At the market, for example, objects are placed in piles of a value of 10 toea; if one is interested in the product, one simply picks up a heap and leaves a coin of 10 toea, which obviates the need for calculations. Wassmann and Dasen (1994) probed Yupno knowledge of arithmetic, amongst others by asking subtractions in the form of bride price problems, e.g., 17 - 9 became "you need 17 pigs to pay a bride price, and you have already given 9 pigs to your prospective father-in-law. How many pigs do you still need?" Subjects could calculate the subtractions by recasting them into additions, in this case, by adding up from 9 and determining how much is needed to reach 17-in other words, they spontaneously figured out a relationship between addition and subtraction. In another experiment, monolingual children who spoke either Warlpiri or Anindilyakwa, two Australian aboriginal languages lacking exact number words, were asked to divide 6 or 9 discs among three toy bears. Despite their unfamiliarity with division, most children successfully solved the problem by using a one-to-one correspondence strategy, giving each toy a disc until all were divided (Butterworth et al., 2008). Several Oksapmin adolescents from a Papua New Guinean culture with a 29-part body-part counting system spontaneously developed an ingenious method for solving additions and subtractions by slightly modifying their body-part counting system. To calculate 7 +5 , they started counting at the body part that denotes 7 , i.e., 'right lower arm'. While counting, they used the words from 1 to 5, i.e., the words from 'right thumb' to 'right pink'. Once this count was completed, they could check where they ended up, which in this case was 'right ear', denoting 12 (Saxe, 1985). These examples, while far from a systematic cross-cultural comparative study, suggest that intuitions about basic arithmetic operations are shared across cultures. Of 
course, there are many cross-cultural differences in the way arithmetic operations are solved, in the base-sizes that are used, and in the way numerals are denoted. Yet to date, no culture has been found where arithmetic deviates widely, e.g., where $2+2$ is consistently taken to equal 5 . Across cultures, simple arithmetic operations like addition and subtraction have the same underlying structure, even though the manner in which results are obtained can vary widely.

\section{Concluding remarks}

In the history of philosophy, mathematics has often served as a paradigmatic example of innate ideas. Although nativism does not figure in current philosophy of mathematics, it is an important guiding principle in developmental psychological investigations of mathematical knowledge. In this paper, we provided a detailed analysis of the claim by Wynn (1992) and other developmental psychologists that knowledge of some principles of arithmetic operations is innate, by spelling out the argumentative structure in detail and by examining some responses to objections against this claim. Our examination of possible relationships between intuitive and formal arithmetic indicates that there are good reasons to think that innate numerical abilities play a significant role in the development of arithmetic competence, even though intuitive number concepts do not correspond to any established set in number theory, and even though children's learning of number does not clearly follow axiomatizations of number. This proposal does not solve the problem of how exactly children go from intuitive numerical skills to arithmetic. A satisfying account of this likely involves both internal and external cognitive factors, such as the use of symbolic representation systems or finger counting as epistemic tools (De Cruz, 2008). Our discussion of arithmetic corroborates the view, defended by early nativist philosophers, that mathematical skills have a cognitive basis, and that this provides a foundation for more formalized mathematical knowledge.

\section{Acknowledgments}

This research is supported by grant $3 \mathrm{H} 070815$ from the Research Foundation Flanders and grant COM07/PWM/001 from Ghent University. We thank Leon Horsten for comments on an earlier version of this paper.

\section{References}

Antell, S.E., Keating, D.P.: 1983, 'Perception of numerical invariance in neonates', Child Development 54, 697-701.

Benacerraf, P.: 1973, 'Mathematical truth', Journal of Philosophy 70, 661-680. Berger, A., Tzur, G., Posner, M.: 2006, 'Infant brains detect arithmetic errors', Proceedings of the National Academy of Sciences USA 103, 12649-12653.

Biro, D., Matsuzawa, T.: 2001, 'Chimpanzee numerical competence: Cardinal and 
ordinal skills', in T. Matsuzawa (ed.), Primate origins of human cognition and behavior, Tokyo: Springer, pp. 199-225.

Brannon, E.M.: 2002, 'The development of ordinal numerical knowledge in infancy', Cognition 83, 223-240.

Butterworth, B., Reeve, R., Reynolds, F., Lloyd, D.: 2008, 'Numerical thought with and without words: Evidence from indigenous Australian children', Proceedings of the National Academy of Sciences USA 105, 13179-13184.

Carey, S.: 2004, 'Bootstrapping and the origin of concepts', Daedalus 133, 59-68.

Cohen, L.B., Marks, K.S.: 2002, 'How infants process addition and subtraction events', Developmental Science 5, 186-212.

Davis, P.J., Hersh, R.: 1981, The mathematical experience, Boston: Birkhauser.

Decock, L.: 2008, 'The conceptual basis of numerical abilities: One-to-one correspondence versus the successor relation', Philosophical Psychology 21, 459473.

De Cruz, H.: 2008, 'An extended mind perspective on natural number representation', Philosophical Psychology 21, 475-490.

De Cruz, H.: 2009, 'An enhanced argument for innate elementary geometric knowledge and its philosophical implications', in B. Van Kerkhove (ed.), New perspectives on mathematical practices. Essays in philosophy and history of mathematics, New Jersey: World Scientific, pp. 185-206.

Dehaene, S., Izard, V., Spelke, E., Pica, P.: 2008, 'Log or linear? Distinct intuitions of the number scale in Western and Amazonian indigene cultures', Science 320, 12171220.

Descartes, R.: 1637 [1988], 'Le discours de la méthode, la dioptrique, les météores et la géometrie', in F. Alquie (ed.), Euvres philosophiques de Descartes, Paris: Classiques Garnier, pp. 549-761.

Feigenson, L., Dehaene, S., Spelke, E.S.: 2004, 'Core systems of number', Trends in Cognitive Sciences 8, 307-314.

Flombaum, J.I., Junde, J.A., Hauser, M.D.: 2005, 'Rhesus monkeys (Macaca mulatta) spontaneously compute addition operations over large numbers', Cognition 97, 315325.

Frank, M.C., Everett, D.L., Fedorenko, E., Gibson, E.: 2008, 'Number as a cognitive technology: Evidence from Pirahã language and cognition', Cognition 108, 819-824.

Gallistel, C.R., Gelman, R.: 2000, 'Non-verbal numerical cognition: From reals to integers', Trends in Cognitive Sciences 4, 59-65.

Gilmore, C.K., McCarthy, S.E., Spelke, E.S.: 2007, 'Symbolic arithmetic knowledge without instruction', Nature 447, 589-591.

Gordon, P.: 2004, 'Numerical cognition without words: Evidence from Amazonia', Science 306, 496-499.

Grabiner, J.V.: 1986, 'Is mathematical truth time-dependent?', in T. Tymoczko (ed.), New directions in the philosophy of mathematics, Boston: Birkhauser, pp. 201-213.

Greiffenhagen, C., Sharrock, W.: 2006, 'Mathematical relativism: Logic, grammar and arithmetic in cultural comparison', Journal for the Theory of Social Behaviour 36, 97 117. 
Haith, M.M.: 1998, 'Who put the cog in infant cognition? Is rich interpretation too costly?', Infant Behavior and Development 21, 167-179.

Halberda, J., Mazzocco, M.M., Feigenson, L.: 2008, 'Individual differences in nonverbal number acuity correlate with maths achievement', Nature 455, 665-668.

Jordan, K.E., Brannon, E.M.: 2006, 'The multisensory representation of number in infancy', Proceedings of the National Academy of Sciences USA 103, 3486-3489.

Kant, I.: 1781 [2005], Critique of pure reason (P. Guyer, A.W. Wood, eds.), Cambridge: Cambridge University Press.

Kobayashi, T., Hiraki, K., Mugitani, R., Hasegawa, T.: 2004, 'Baby arithmetic: One object plus one tone', Cognition 91, B23-B34.

Koechlin, E., Dehaene, S., Mehler, J.: 1998, 'Numerical transformations in five-monthold human infants', Mathematical Cognition 3, 89-104.

Laurence, S., Margolis, E.: 2005, 'Number and natural language', in P. Carruthers, S. Laurence, S. Stich (eds.), The innate mind. Structure and contents, Oxford: Oxford University Press, pp. 216-235.

Le Corre, M., Carey, S.: 2007, 'One, two, three, four, nothing more: An investigation of the conceptual sources of the verbal counting principles', Cognition 105, 395-438.

Leibniz, G.W.: 1765 [2001], New essays on human understanding (P. Remnant, J.F. Bennett, eds.), Cambridge: Cambridge University Press.

Mameli, M., Bateson, P.: 2006, 'Innateness and the sciences', Biology and Philosophy 21, 155-188.

McCrink, K., Wynn, K.: 2004, 'Large-number addition and subtraction by 9-month-old infants', Psychological Science 15, 776-781.

Meck, W.H., Church, R.M.: 1983, 'A mode control model of counting and timing processes', Journal of Experimental Psychology: Animal Behavior Processes 9, 320334.

Piaget, J.: 1952, The child's conception of number, New York: Norton.

Plato: ca. 380 B.C. [2000], 'Meno', in S.M. Cahn (ed.), Exploring philosophy. An introductory anthology, New York: Oxford University Press, pp. 117-151.

Resnik, M.D.: 1982, 'Mathematics as the science of patterns: epistemology', Noûs 16, 95-105.

Rips, L., Bloomfield, A., Asmuth, J.: 2008, 'From numerical concepts to concepts of number', Behavioral and Brain Sciences 31, 623-642.

Samuels, R.: 2002, 'Nativism in cognitive science', Mind and Language 17, 233-265.

Samuels, R.: 2004, 'Innateness in cognitive science', Trends in Cognitive Sciences 8, 136-141.

Saxe, G.B.: 1985, 'Effects of schooling on arithmetic understandings: Studies with Oksapmin children in Papua New Guinea', Journal of Educational Psychology 77, 503513.

Shapiro, S.: 1997, Philosophy of mathematics: Structure and ontology, Oxford: Oxford University Press.

Siegler, R.S., \& Booth, J.L.: 2004, 'Development of numerical estimation in young children', Child Development 75, 428-444.

Spelke, E.S., Kinzler, K.D.: 2007, 'Core knowledge', Developmental Science 10, 89-96. 
Thune, C.E.: 1978, 'Numbers and counting in Loboda: An example of a non-numerical oriented culture', Papua New Guinea Journal of Education 14, 69-80.

Thurston, W.: 2006, 'On proof and progress in mathematics', in R. Hersh (ed.), 18 unconventional essays on the nature of mathematics, New York: Springer, pp. 37-55.

Tudusciuc, O., Nieder, A.: 2007, 'Neuronal population coding of continuous and discrete quantity in the primate posterior parietal cortex', Proceedings of the National Academy of Sciences USA 104, 14513-14518.

Uller, C., Jaeger, R, Guidry, G., Martin, C.: 2003, 'Salamanders (Plethodon cinereus) go for more: Rudiments of number in an amphibian', Animal Cognition 6, 105-112.

Venkatraman, V., Ansari, D., Chee, M.W.L.: 2005, 'Neural correlates of symbolic and non-symbolic arithmetic', Neuropsychologia 43, 744-753.

Wassmann, J., Dasen, P.R.: 1994, 'Yupno number system and counting', Journal of Cross-Cultural Psychology 25, 78-94.

Whalen, J., Gallistel, C., Gelman, R.: 1999, 'Nonverbal counting in humans: The psychophysics of number representation', Psychological Science 10, 130-137.

Wynn, K.: 1992, 'Addition and subtraction by human infants', Nature 358, 749-750.

Author affiliations:

Helen De Cruz (corresponding author): Centre for Logic and Analytical Philosophy, Katholieke Universiteit Leuven, Kardinaal Mercierplein 2, 3000 Leuven, Belgium, Helen.DeCruz@hiw.kuleuven.be

Johan De Smedt: Department of Philosophy and Ethics, Ghent University, Blandijnberg 2, 9000 Ghent, Belgium, johan.desmedt@ugent.be 